Graduate Students,

\title{
A systematic review of pharmacist input in the screening, management and prevention of metabolic syndrome
}

\section{Rana Al Adawi ${ }^{1}$, Derek Stewart ${ }^{2}$, Cristin Ryan ${ }^{3}$, Antonella Tonna ${ }^{2}$.}

Hamad Medial Corporation ${ }^{1}$, Robert Gordon University2, Trinity College Dublin ${ }^{3}$.

Available from: http://www.crd.york.ac.uk/PROSPERO/display record.php?ID=CRD42018089862

\section{Background:}

Metabolic syndrome (MetS) is a cluster of factors that increase the risk of cardiovascular disease and include diabetes, abdominal obesity, elevated triglyceride\$, low high-density lipoprotein cholesterol and high blood-pressure. A patient is considered to have MetS if 3 out of 5 factors are present 1 .

\section{Purpose:}

To critically appraise, synthesise, and present the available evidence on: the types and impact of pharmacist input in MetS, to characterize the populations who would benefit most and to describe facilitators and barriers.

\section{Methods:}

* Search string: Pharm, "Metabolic syndrome*”, "Syndrome X", "Insulin resistance syndrome*", "Dysmetabolic syndrome waist"”, "Obesity syndrome*”, "Metabolic Cardiovascular Syndrome", "Reaven Syndrome X", "Atherothrombogenic syndrome"

- Databases: Medline, Cumulative Index of Nursing and Allied Health Literature (CINAHL), International Pharmaceutical Abstracts (IPA), Cochrane Database of Systematic Reviews

- Included studies: Peer-reviewed papers published in English from 2008

Papers assessed: By two reviewers for methodological quality

Critically appraised: Data extracted using standardized tools ${ }^{2}$

\section{Results:}

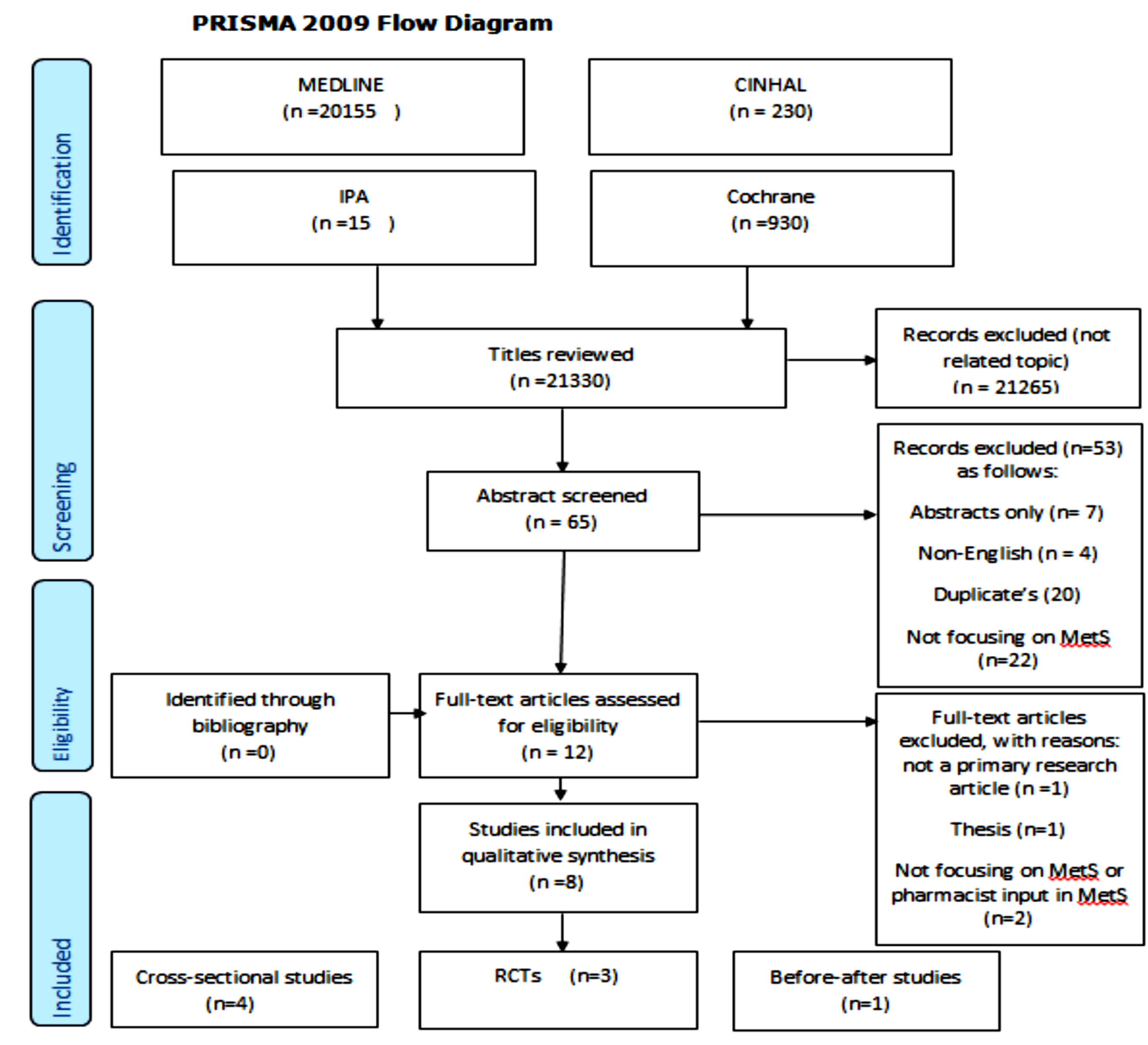

Figure 1 - Search flowchart as an adapted PRISMA diagram ${ }^{3}$

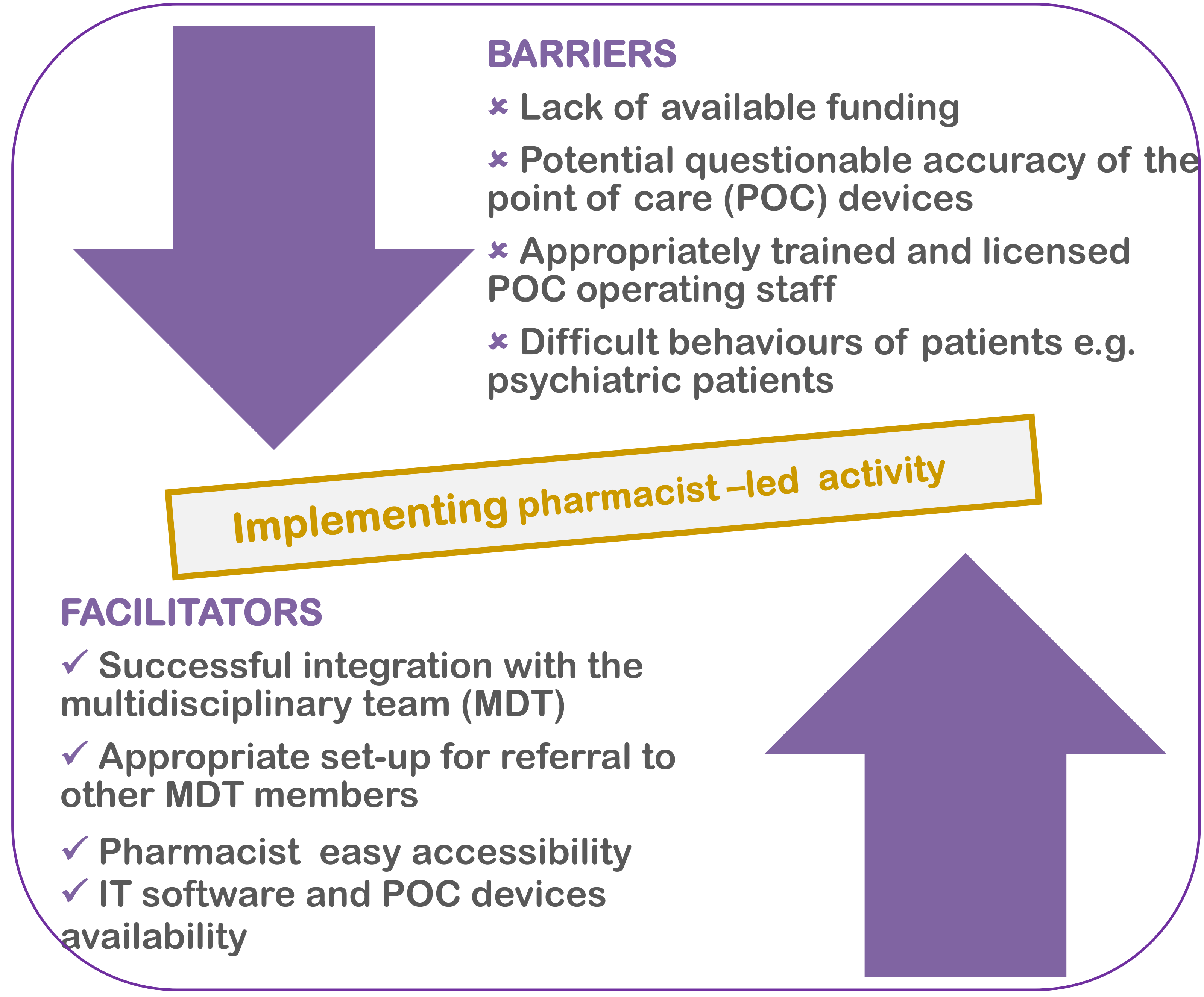

Figure 3- Some facilitators and barriers of pharmacist-led implementation
Children and adolescent

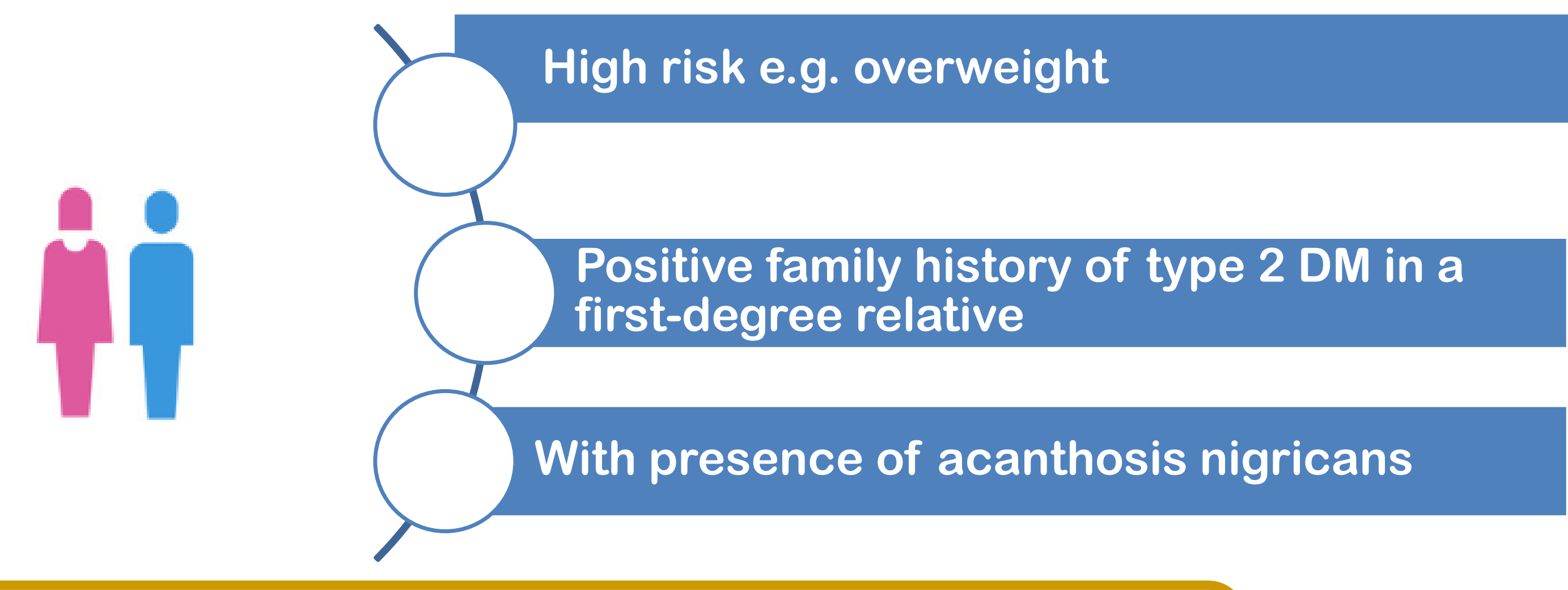

Populations that would benefit the most

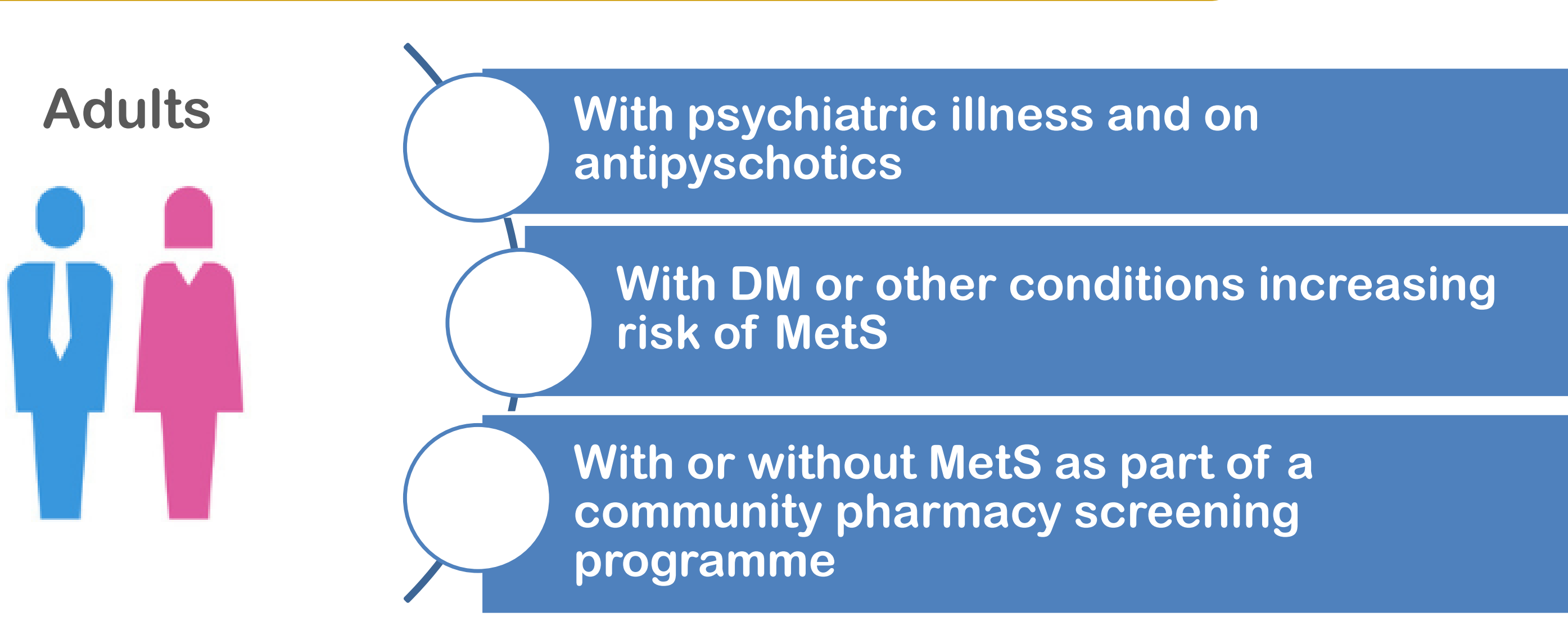

Figure 2 - The population who would benefit the most from the pharmacist input in Mets

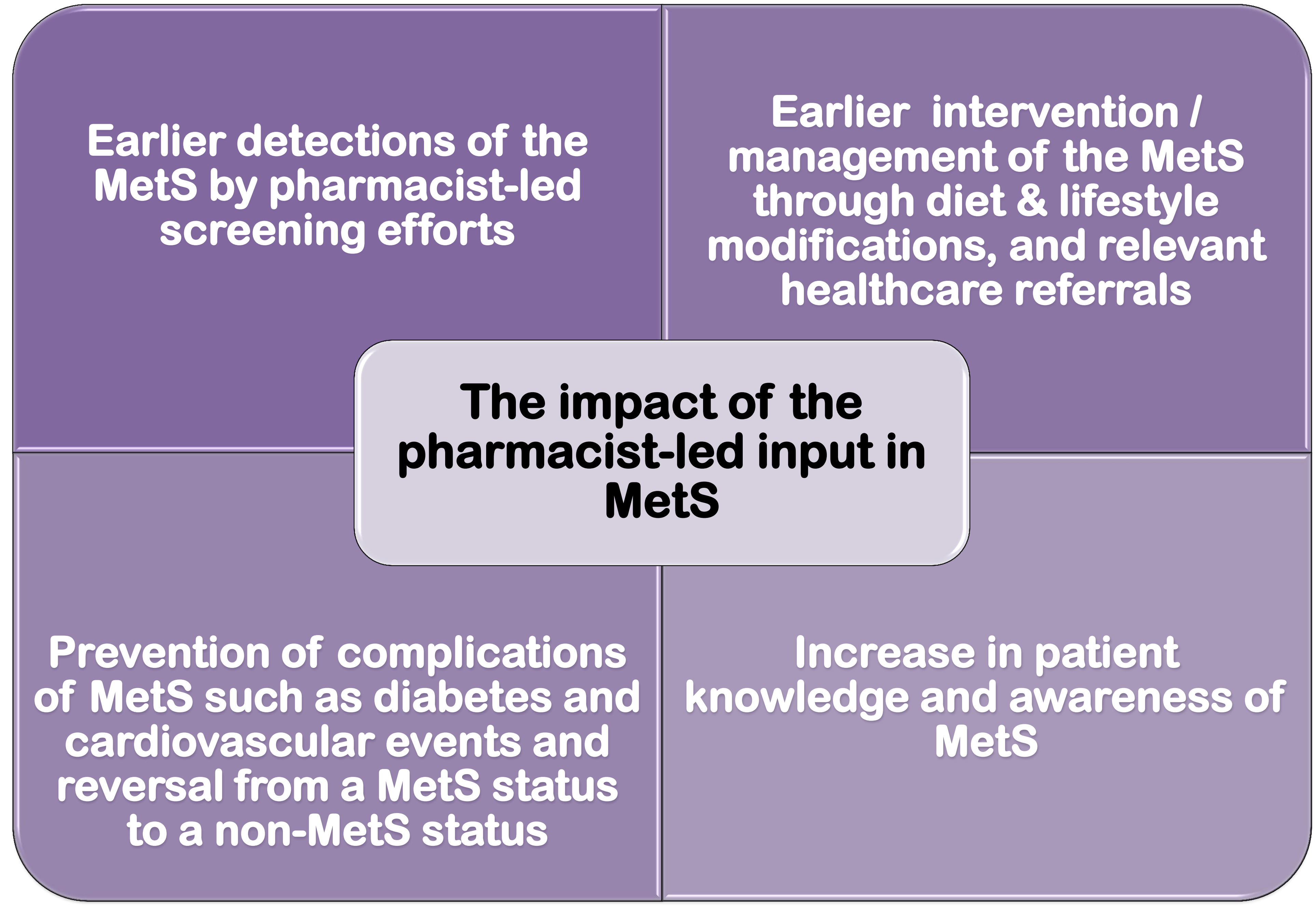

Figure 4 - Impact of the pharmacist input
Pharmacists can effectively participate in the screening, prevention and management of Mets in different populations and settings to enhance patient's care.

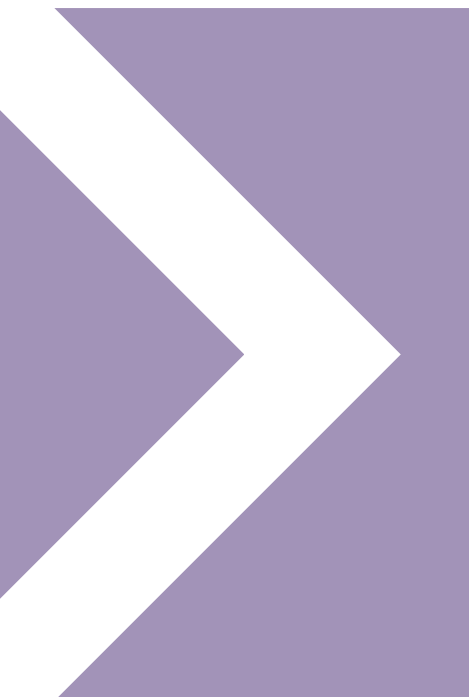

Further research is warranted to determine the clinical and economic impact and describe the facilitators and barriers of implementing such a program.
Disclosure: None of the authors of this study have to disclose any possible financial or personal relationships with commercial entities that may have a direct or indirect interest in the subject matter of this study.

Correspondence to: Rahmed4@hamad.qa

\section{References:}

1.Alberti, G. Zimmet, P. Shaw, J. and Grundy, S.M., 2006. The IDF consensus worldwide definition of the metabolic syndrome. Brussels: International Diabetes Federation, 23(5), 469-80.

2.Nhlbi.nih.gov. (2018). Study Quality Assessment Tools | National Heart, Lung, and Blood Institute (NHLBI). [online] Available at: $h$ ttps://www.nhlbi.nih.gov/health-topics/study-quality-assessment-tools [Accessed 10 Sep. 2018].

3.Moher D, Liberati A, Tetzlaff J, Altman DG. Preferred reporting items for systematic reviews and meta-analyses: the PRISMA statement. PLoS Medicine. 2009; 6(7):e1000097. 